\title{
Long-Term Functionality of Transversal Intraneural Electrodes Is Improved By Dexamethasone Treatment
}

\author{
N. de la Oliva ${ }^{1}$, J. del Valle ${ }^{1,2}$, I. Delgado-Martínez ${ }^{1}$, M. Mueller ${ }^{3}$, Student Member IEEE, T. \\ Stieglitz ${ }^{3,4,5}$, Senior Member IEEE, Xavier Navarro ${ }^{1 *}$
}

\begin{abstract}
Neuroprostheses aimed to restore lost functions after a limb amputation are based on the interaction with the nervous system by means of neural interfaces. Among the different designs, intraneural electrodes implanted in peripheral nerves represent a good strategy to stimulate nerve fibers to send sensory feedback and to record nerve signals to control the prosthetic limb. However, intraneural electrodes, as any device implanted in the body, induce a foreign body reaction (FBR) that results in the tissue encapsulation of the device. The FBR causes a progressive decline of the electrode functionality over time due to the physical separation between the electrode active sites and the axons to interface. Modulation of the inflammatory response has arisen as a good strategy to reduce the FBR and maintain electrode functionality. In this study transversal intraneural multi-channel electrodes (TIMEs) were implanted in the rat sciatic nerve and tested for 3 months to evaluate stimulation and recording capabilities under chronic administration of dexamethasone. Dexamethasone treatment significantly reduced the threshold for evoking muscle responses during the follow-up compared to saline-treated animals, without affecting the selectivity of stimulation. However, dexamethasone treatment did not improve the signal-to-noise ratio of the recorded neural signals. Dexamethasone treatment allowed to maintain more working active sites along time than saline treatment. Thus, systemic administration of dexamethasone appears as a useful treatment in chronically implanted animals with neural electrodes as it increases the number of functioning contacts of the implanted TIME and reduces the intensity needed to stimulate the nerve.
\end{abstract}

Index Terms - dexamethasone, foreign body reaction, intraneural electrode, neuroprosthesis, stimulation.

1 Institute of Neurosciences, Department of Cell Biology, Physiology and Immunology, Universitat Autònoma de Barcelona, and Centro de Investigación Biomédica en Red en Enfermedades Neurodegenerativas (CIBERNED), Bellaterra, Spain.

2 Catalan Institute of Nanoscience and Nanotechnology (ICN2), CSIC and BIST, Campus UAB, Bellaterra, Spain.

3 Laboratory for Biomedical Microtechnology, Department of Microsystems Engineering IMTEK, Albert-Ludwig-University Freiburg, Freiburg, Germany

4 BrainLinks-BrainTools Cluster of Excellence, Albert-Ludwig-University Freiburg, Freiburg, Germany

5 Bernstein Center Freiburg, Albert-Ludwig-University Freiburg, Freiburg, Germany.

*X.N. Faculty of Medicine, Universitat Autònoma de Barcelona, 08193 Bellaterra, Spain (correspondence e-mail: Xavier.navarro@uab.cat).

\section{INTRODUCTION}

$\mathrm{T}$ he interface of neuroprosthetic systems intended to substitute an amputated limb is based in the stimulation of sensory axons to evoke sensory feedback and in the recording of motor nerve signals to control the prosthetic device. Peripheral nerve electrodes are a key component of neuroprostheses, as they are the active part that will interact with the nervous tissue [1]. Despite the different designs, most nerve electrodes consist of a polymer substrate where metal active sites are embedded to deliver current to stimulate nerve fibers or to record nerve signals. Therefore, the relative position and distance to the nerve fibers will determine the intensity needed to stimulate the axons and the quality of the recorded signals. Among the different designs developed, intraneural electrodes, such as the transversal intrafascicular multichannel electrode (TIME), have shown good capabilities for selective stimulation and recording in comparison to extraneural electrodes [2].

However, studies in human subjects and animal experiments have shown a progressive decline in the intraneural electrode functionality over time [3]-[5]. This has been attributed, at least in part, to the foreign body reaction (FBR) triggered after the implantation of the device [6], [7]. The FBR against an intraneural electrode is characterized by an initial inflammatory phase, in which polymorphonuclear cells and monocytes/macrophages infiltrate the tissue from 1 day after the implant. This first phase induces a later antiinflammatory and tissue-remodeling phase from 8 weeks postimplant, mainly orchestrated by fibroblasts [8]-[11]. This FBR results in tissue encapsulation of the electrodes, leading to a physical separation of the active sites from the nerve fibers [9], [11] and an increase in the impedance [10], [12]. Since the relative position and distance to the nerve fibers is critical to determine the intensity needed to stimulate the axons and the quality of the recorded signals, the capsule tissue around the electrode clearly hampers its functionality over time.

Different strategies have been pursued to improve the longterm functional outcome of implanted neural electrodes, including the use of different polymers as a substrate of the electrode [13]-[16], diverse surface coatings [17]-[19] and pharmacological modulators of the FBR [20], [21]. One of the most used drugs to modulate the FBR is dexamethasone, a 
glucocorticoid known for its anti-inflammatory and immunedepressant effects [22]. Dexamethasone administration has shown good results in reducing the inflammatory reaction to implanted electrodes in the central nervous system [23], [24] as well as improving brain recordings [25]. Some studies have applied these strategies to peripheral nerve electrodes, as for example, using dexamethasone-releasing cuff [26] or dexamethasone-loaded multichannel [27] electrodes. However, these studies did not take into account the timewindow in which such modulators may be effective or if modulation of the FBR has, indeed, positive effects on the functional performance of the electrodes for stimulation and recording. Systemic administration of drugs for reducing the FBR may allow for a better control of dosage and timing without modifications in the design of current nerve electrodes [28]. Thus, this study aims to evaluate if dexamethasone treatment improves the long-term functional outcome of TIME electrodes implanted in the rat sciatic nerve.

\section{Material AND MethodS}

\section{A. Surgical Procedures and Drug Administration}

All animal experiments conducted in this study were performed according to protocols approved by the Ethical Committee of the Universitat Autònoma de Barcelona in accordance with the European Communities Council Directive 2010/63/EU.

The electrode used was a transversal intrafascicular multichannel electrode (TIME version $3 \mathrm{H}$; developed by the Department of Microsystems Engineering-IMTEK, University of Freiburg, Germany) [29], [30]. It contains seven active sites made of iridium oxide at each arm of the device, with a diameter of $80 \mu \mathrm{m}$ in one arm and a diameter ranging from 20 to $60 \mu \mathrm{m}$ in the other. In this study, only the active sites of 80 $\mu \mathrm{m}$ at one arm were evaluated. The TIME electrodes were implanted in the sciatic nerve of female Sprague-Dawley rats $(\mathrm{n}=12,220 \pm 20 \mathrm{~g})$. Animals were anesthetized with ketamine and xylazine $(90 / 10 \mathrm{mg} / \mathrm{kg}$, i.p.) and the sciatic nerve was surgically exposed at the midthigh and freed from adherences to surrounding tissues. Following the same procedure as in [29], the TIME was transversally inserted across the sciatic nerve with the help of a straight needle attached to a 10-0 loop thread. All the process was monitored under a dissection microscope to ensure the correct position of the active site inside the nerve fascicles (Fig. 1A). Since the TIME used was originally designed for human nerves, in most cases, only 2-3 of seven contacts were positioned within the rat sciatic nerve at the same time due to dimension mismatch. Then, the electrode tip was fixed to the closest muscle with a 10-0 suture to avoid the electrode sliding from the nerve and reduce motion. The fixation flaps in the arm of the TIME3H were not used to fix it to the epineurium, as done in human nerve implants [3], to avoid nerve damage. This fixation was chosen in order to assess functional stability over time, i.e. to maintain the electrode inside the nerve, but without further damaging it. The ceramic interconnector, on which the TIME tracks are connected to wires, was fixed subcutaneously over the gluteus muscles to reduce tethering forces on the electrode. The wires were routed inside a thin silicon tube subcutaneously and soldered to a Circular Omnetics connector (Nano series) that was fixed with a custom-made plastic base on the back of the animal to facilitate the connection during the electrophysiological tests and covered with a metal protection cap (Fig. 1B). The muscles and the skin were closed with sutures and the wounds disinfected with povidone-iodine. After the surgical procedure, animals were left to recover in warm pads. During the follow-up, they were housed at $22 \pm 2^{\circ} \mathrm{C}$ under a 12:12 h light cycle with food and water access ad libitum.
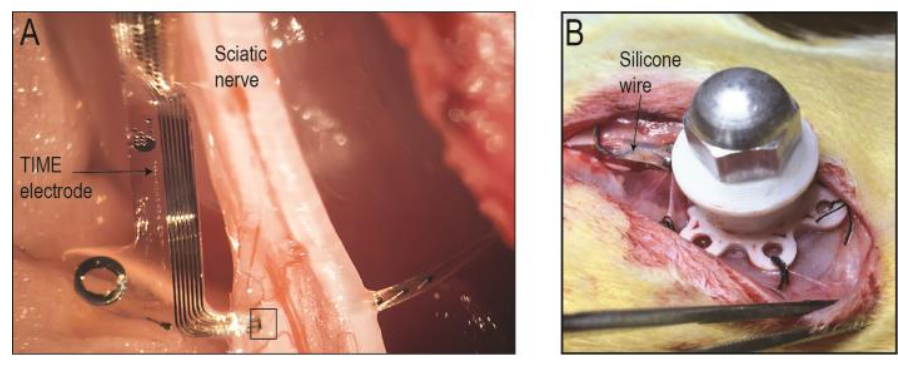

Figure 1. Surgical implant of a TIME in the rat sciatic nerve. (A) Photograph of the TIME inserted across tibial and peroneal fascicles of the sciatic nerve. The tip of the TIME was sutured to the close muscle to avoid motion. Active site 1 (square) is almost out but still within the nerve. Scale bar $=1 \mathrm{~cm}$. (B) View of the back of the animal with the subcutaneous wires and the plastic base made to house the Omnetics connector. A metal cup was used to prevent damage to the connector.

Half of the rats were administered daily with dexamethasone ( $\mathrm{n}=6,200 \mu \mathrm{g} / \mathrm{kg}$ subcutaneous; Merck) from two days before the surgery to ensure systemic levels and during all the follow-up. The other half received injections of the same volume of saline vehicle. After 12 weeks postimplant, animals were deeply anesthetized with an overdose of pentobarbital, transcardially perfused with $4 \%$ paraformaldehyde in phosphate buffer and the sciatic nerve including the implanted device harvested for histological analysis.

\section{B. Histological Studies}

The nerve segment including the intraneural electrode was embedded in paraffin, and transverse sections (10 $\mu \mathrm{m}$ thick) were cut, mounted on silane-coated slides and dried overnight. To identify the location of TIME electrode inside the nerve and to evaluate the presence of foreign body giant cells (FBGCS) and the encapsulating tissue, sections were deparaffinized and stained, first overnight with standard luxol fast blue (LFB) for myelin visualization, and then with hematoxylin Harris solution (Fluka, Sigma) for $7 \mathrm{~min}$, washed with water and stained with Eosin Y (Merck Millipore) for 3 min. Sections were dehydrated with series of graded ethanol rinses and mounted with DPX (Sigma). For encapsulation measurement, the area and the surface occupied by the encapsulating tissue around the electrode were measured, and a mean thickness of the capsule was obtained. The number of foreign body giant cells (FBGCs) was counted in each section (4 sections/animal, $\mathrm{n}=6 /$ group). 


\section{Nerve Stimulation Protocol}

To assess the stimulation performance of the implanted TIMEs, biphasic rectangular current pulses with a width of $100 \mu \mathrm{s}$ and an intensity up to $1 \mathrm{~mA}$ were delivered (Stimulator DS4, Digitimer) through each one of the active sites against a small needle electrode placed near the nerve. The compound muscle action potentials (CMAP) were recorded from gastrocnemius medialis (GM), tibialis anterior (TA) and plantar interossei (PL) muscles using small needle electrodes placed in each muscle [2]. The CMAPs were amplified (P511AC amplifiers, Grass), band-pass filtered (3 Hz to 3 $\mathrm{kHz}$ ) and digitized with a Powerlab recording system (PowerLab16SP, ADInstruments) at $20 \mathrm{kHz}$. The amplitude of each CMAP ( $M$ wave) was measured peak to peak and normalized to the maximum CMAP amplitude obtained in each experiment by stimulation of the sciatic nerve with a stainless-steel needle electrode. For each active site, the threshold current of stimulation that elicited a 5 and $95 \%$ of the maximum CMAP was determined. The active site with the lowest threshold value in each electrode (best AS) was used for data analysis and comparison between groups (1AS/animal, $n=6$ at day 0,7 and 14 and $n=5$ at day 30 for Dex group and $n=6$ at day 0 and $n=4$ at day 7,14 and 30 for Saline group). To measure the electrode displacement over time, the best active site from each TIME at each time point was determined, the difference with respect to the previous position calculated and the result multiplied by the distance between active sites $(400 \mu \mathrm{m})$. Finally, the selectivity index (SI) was calculated to quantify the specific activation of a single muscle among the set of three muscles (GM, TA, PL) when stimulating from each active site, as previously described [2], [31] and the maximum SI (SImax) from each TIME for each muscle was used.

\section{Nerve Signals Recording Protocol}

To assess the recording capabilities of TIME electrodes over time, two different protocols were performed as previously described [32]. First, the compound nerve action potentials (CNAPs) were recorded from the TIME following electrical stimulation of the distal tibial nerve. Rectangular pulses of $100 \mu \mathrm{s}$ and up to $5 \mathrm{~mA}$ (DS4, Digitimer) were delivered using two small needles inserted on the medial side of the paw. Recordings were made from each active site of the TIME against the counter electrode in the ribbon part of the TIME (outside the nerve), with a small needle electrode subcutaneously inserted as ground. The CNAP amplitude recorded from each site was measured peak to peak. The maximum amplitude recorded from each TIME was analyzed for comparison between groups ( $\mathrm{N}$ per groups as detailed in the nerve stimulation part). Second, neural activity evoked by scratching with a blunt plastic probe the plantar surface of the paw (10 repetitions) was recorded from each active site [33]. Signals were amplified x5000, band-pass filtered (between $300 \mathrm{~Hz}$ and $10 \mathrm{kHz}$ ) and fed to a power-line noise eliminator (Hum Bug, Quest Scientific), then digitized at $20 \mathrm{kHz}$ and recorded with Chart software (PowerLab System, ADInstruments). The total power of the recorded signals and the noise (no stimulus applied) was obtained after applying the short-time Fourier transform with a window of $1 \mathrm{~ms}$, and an overlap of $87.5 \%$. Only the best recording active site in each TIME was used to calculate the signal-to-noise ratio (SNR), as the ratio between the mean of the total power when the stimuli are applied and the mean of the total power when there are no stimuli applied.

Animal body temperature was maintained constant using a thermostatic heating pad during the electrophysiological tests. Both stimulation and recording protocols were performed acutely ( 0 days, 30 minutes after the implantation) and at 7 , $14,30,60$ and 90 days after implant or until failure of the electrode.

\section{E. Statistical Analysis}

Results are expressed as the average \pm SEM. Normality of distribution was tested by the Shapiro-Wilk test. Differences between groups or times in normally distributed data were analyzed by two-way ANOVA followed by Sidak's post hoc tests. For non-normal variables the analysis was performed with the Kruskal-Wallis test followed by Dunn's test. Statistical significance was considered at $\mathrm{p}<0.05$. The GraphPad Prism software was used for all statistical analyses.

\section{RESULTS}

Animals implanted with TIME electrodes and administered with dexamethasone or saline were followed for 3 months to evaluate electrode functionality. Unfortunately, the wiring system got damaged before the final date in some cases. Therefore, the comparison between the two groups with respect to the electrophysiological results was made up to 1 month of implantation only (subchronic time-point, [34]), whereas histology was performed in all animals at 3 months (chronic time-point).

\section{A. Nerve Stimulation Results over Time}

The current needed to achieve $5 \%$ of the maximal CMAP (5\% threshold) for each active site in each TIME implanted showed variations over time depending on the active site (Fig. 2A). For contacts located inside the nerve immediately after implantation, the $5 \%$ threshold averaged $70 \pm 10 \mu \mathrm{A}$ in the saline-treated group and $60 \pm 9 \mu \mathrm{A}$ in the dexamethasonetreated group ( $\mathrm{n}=6$ per group), whereas half of the active sites were outside the nerve and had 5\% threshold above $200 \mu \mathrm{A}$. Interestingly, the active site with the lowest threshold changed during the follow-up in each case, indicating a relative motion from the original position (at day 0) of the implanted electrodes (Fig. 2A-B) and suggesting a dynamic integration of the electrode within the nerve rather than a fixed implant, that could damage the tissue with its rigidity. Thus, we quantified the displacement of the electrodes with respect to its original position for the two groups. Saline-treated animals presented longer displacement of the electrode than the dexamethasone-treated group $(1400 \pm 200 \mu \mathrm{m}$ vs $720 \pm 366$ $\mu \mathrm{m}$ ) during the first week of follow-up, equating to the shift of 2 or 3 active sites Differences, however, did not reach statistical significance ( $p>0.05$, Kruskal-Wallis test). In fact, 
the position of the electrodes in animals treated with dexamethasone was more stable over time $(400 \pm 179 \mu \mathrm{m}$ and $160 \pm 98 \mu \mathrm{m}$ after 14 and 30 days, respectively) than the electrodes in the saline-treated group $(300 \pm 191 \mu \mathrm{m}$ and 500 $\pm 252 \mu \mathrm{m}$ after 14 and 30 days, respectively) with respect to the previous position (Fig. 2B).

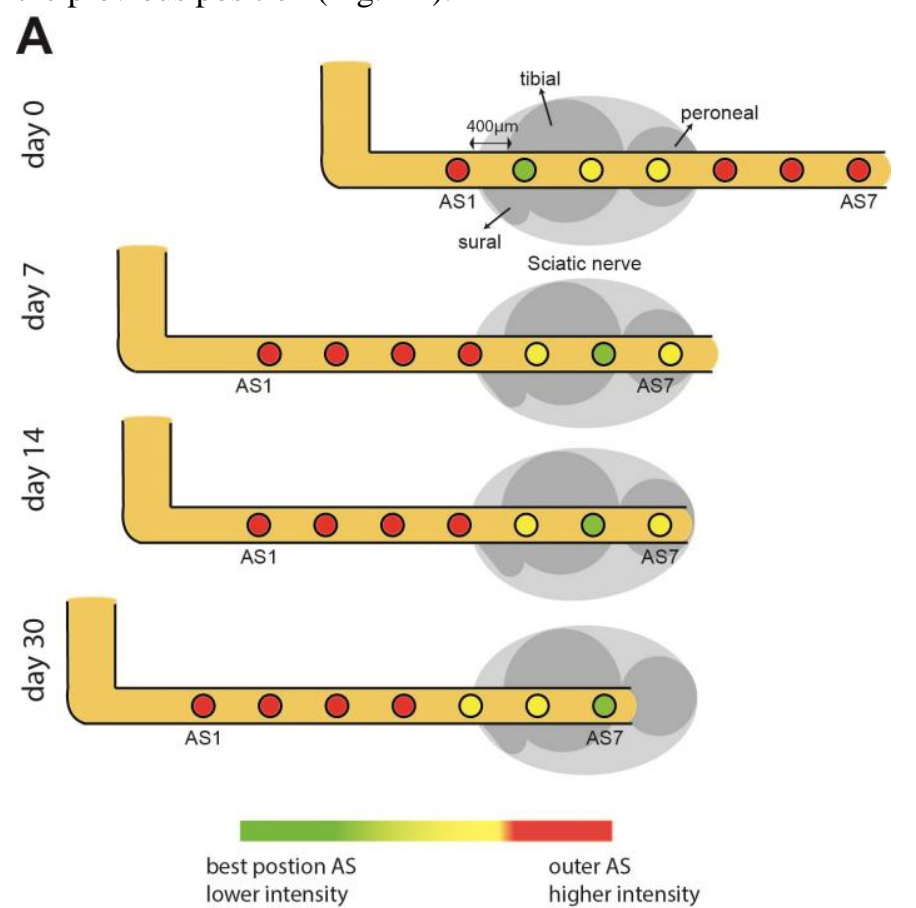

B

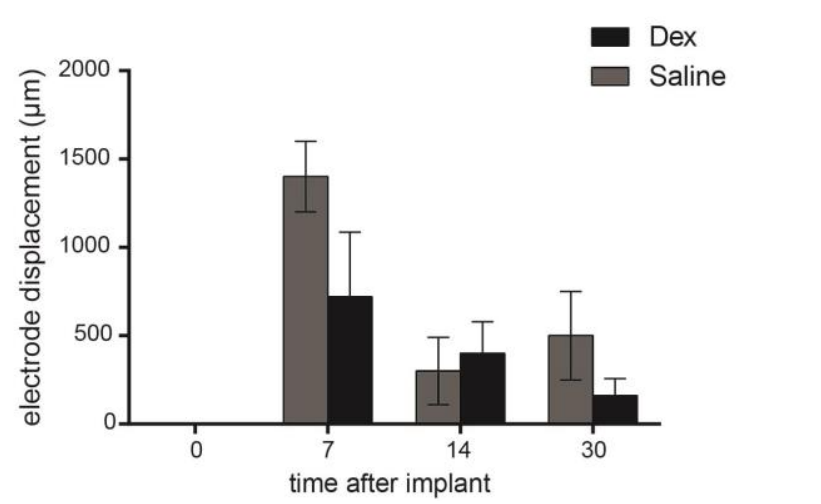

Figure 2. Changes in stimulation threshold and active sites position within the sciatic nerve over time. (A) Schematic view of a given TIME implanted in the fascicles of the sciatic nerve and its shift over time, as derived from values found in the stimulation protocol. Changes in the active site (AS) position correspond to changes in stimulation threshold. (B) Quantification of the displacement of implanted TIMEs in saline- and dexamethasone-treated groups over time. Each column corresponds to the mean change with respect to the previous tested time.

Because of the relative displacement, we used the best active site (i.e., the one with the lowest threshold value which indicates the best position inside the nerve) for each muscle in each electrode for comparison between dexamethasone and saline-treated groups. All animals showed a clear increase in the threshold current needed for muscle activation, at 5\% and 95\% of maximal CMAP, during the first week of follow-up, with later stabilization at 14 and 30 days after implant $(\mathrm{p}<0.05)$. However, rats receiving dexamethasone had significantly lower values for GM and TA muscles in comparison with the saline-treated group $(\mathrm{p}<0.05)$ (Fig. 3), indicating that the treatment limited the initial increase in tissue resistance. The treated group still maintained lower values after 1 month of implant (Fig.3).
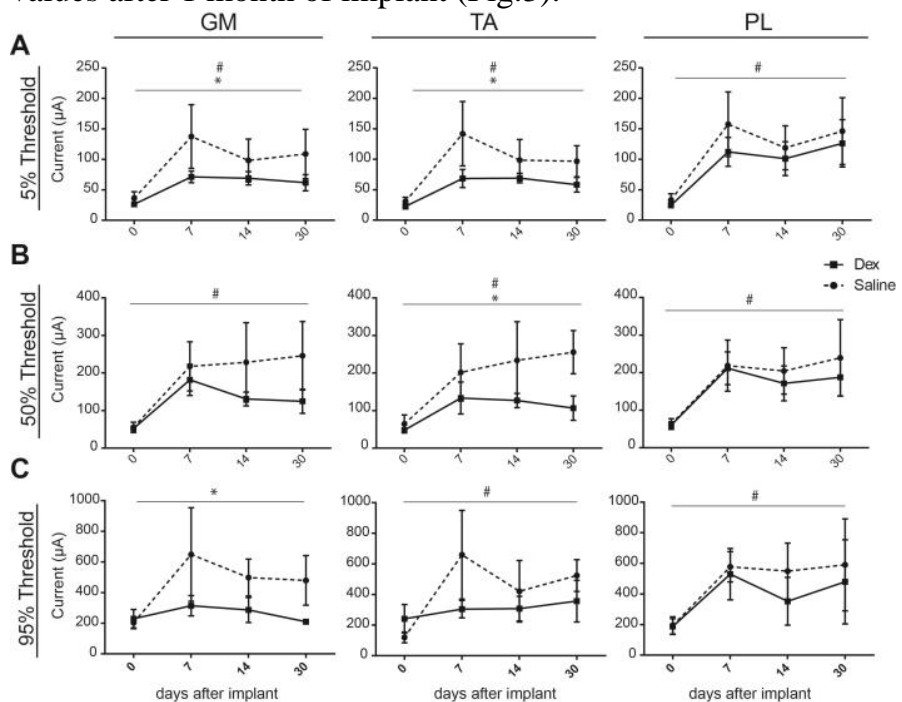

Figure 3. Values of stimulation current needed to elicit (A) $5 \%$, (B) $50 \%$ and (C) $95 \%$ of the maximum CMAP amplitude of gastrocnemius (GM), tibialis anterior (TA) and plantar interossei (PL) muscles in saline and dexamethasone treated groups over time. Two-way ANOVA, ${ }^{*} \mathrm{p}<0.05$ vs saline-treated group, $\# \mathrm{p}<0.05$ vs time.

Additionally, the number of functioning active sites able to evoke CMAPs in the muscles recorded decreased over time for both groups. However, there were more functioning sites during all the follow-up in the dexamethasone-treated than in the control group, although the difference was not significant ( $p>0.05$, Kruskal-Wallis test, Fig. 4). 
A

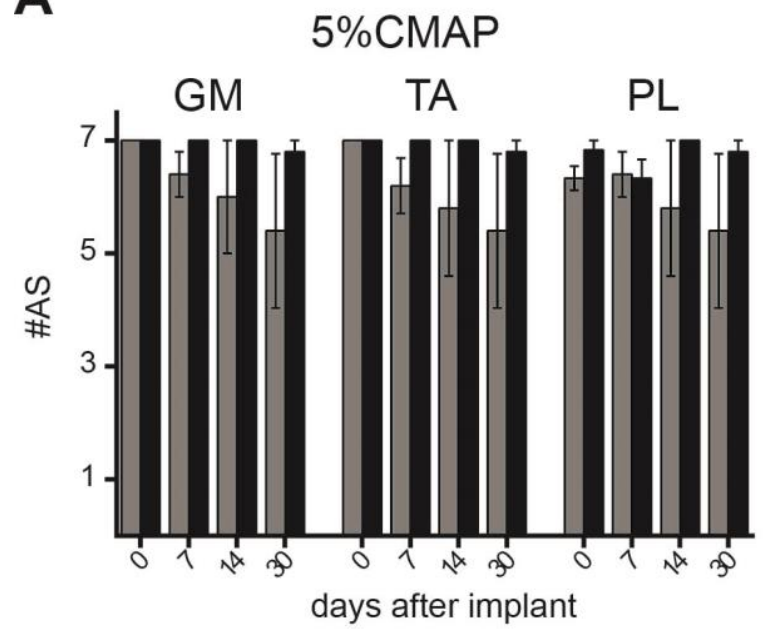

B

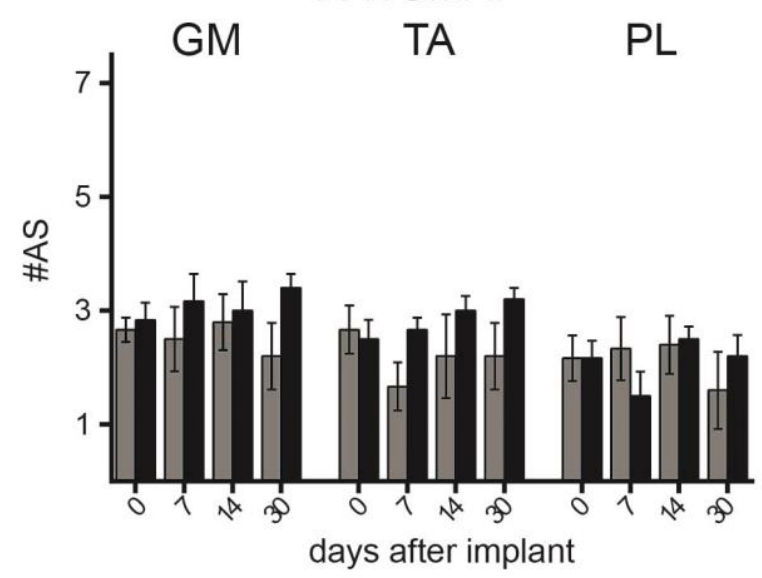

C

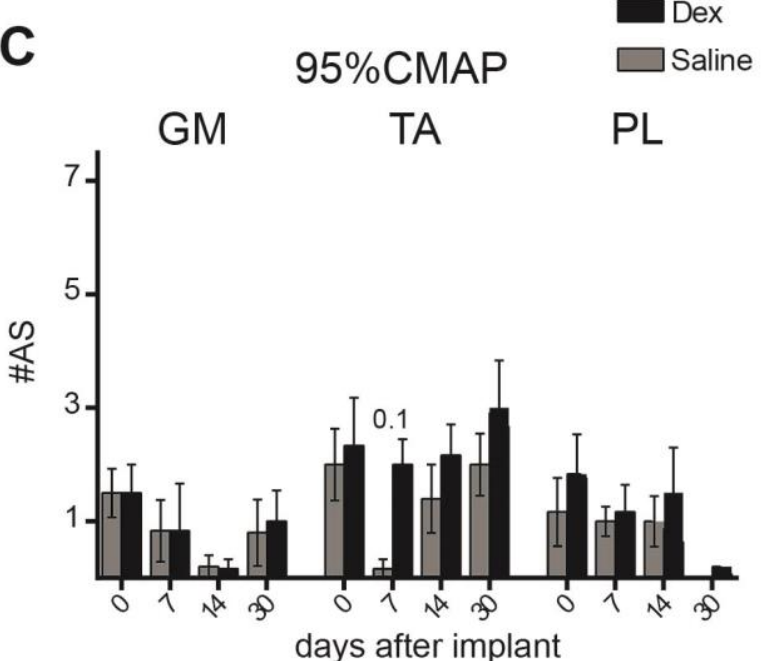

Figure 4. Number of working active sites (AS) in the TIME that were able to evoke a (A) $5 \%$, (B) $50 \%$ and (C) $95 \%$ of the maximum CMAP amplitude of gastrocnemius (GM), tibialis anterior (TA) and plantar interosseus (PL) muscles in saline and dexamethasone treated groups at different time points..

The selectivity of stimulation for the three different muscles tested innervated by the sciatic nerve ranged between 0.56 and 0.9. The SImax was maintained during the follow-up for GM (Fig. 5A) and TA (Fig. 5B) muscles, whereas it decreased from 0 to 30 days after the implant for the PL muscle, without differences between both groups (Fig. 5C).
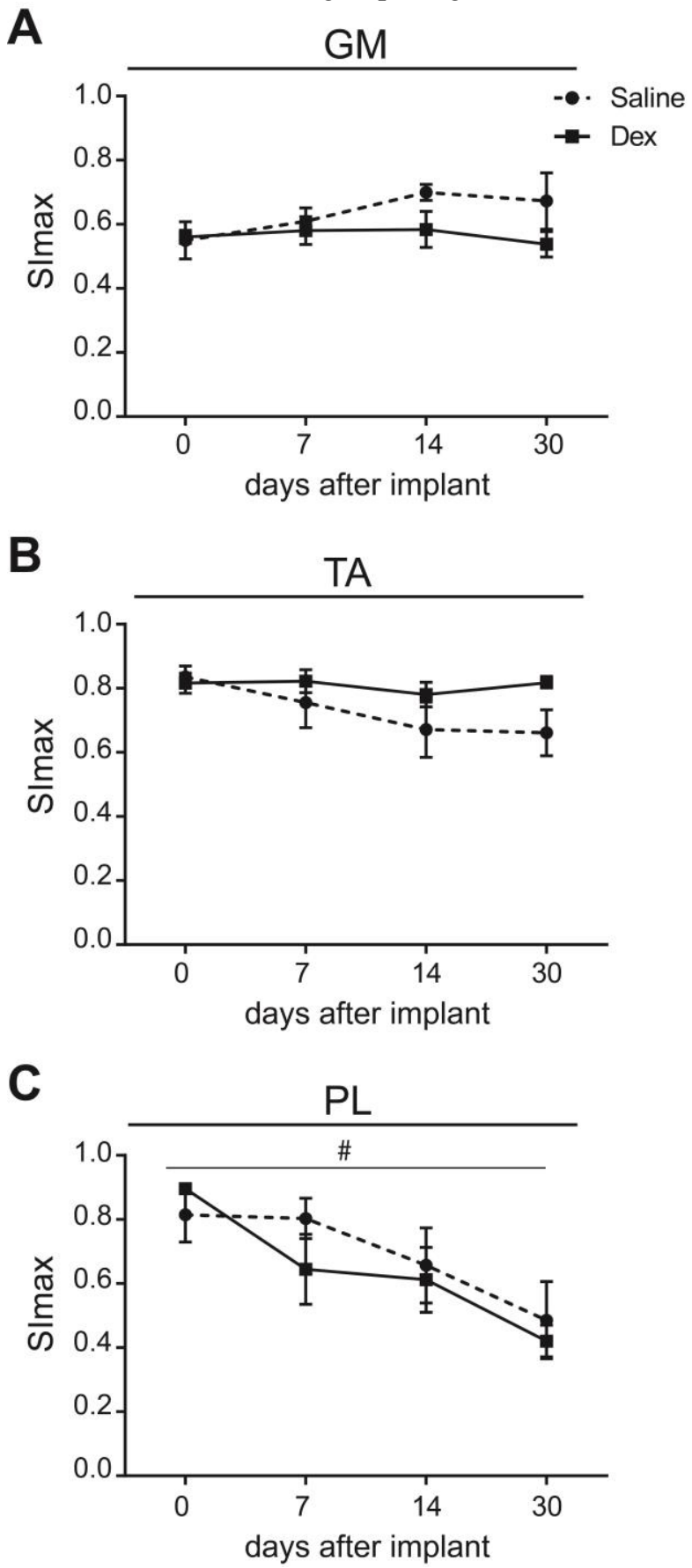

Figure 5. Maximal selectivity index (SImax) for gastrocnemius (GM), tibialis anterior (TA) and plantar interossei (PL) muscles over time in saline and dexamethasone treated groups. Two-way ANOVA, \#p<0.05 vs time.

\section{B. Nerve signal recording results}

The maximum amplitude of the CNAP, elicited by electrical stimulation of the distal tibial nerve and recorded from active sites in the TIME, decreased over time in both groups (Fig. 6A). Dexamethasone treatment allowed recording CNAPs of slightly higher amplitudes, yet not significantly than in control rats during the first two weeks of follow-up (Fig. 6A). Electroneurographic recordings were performed during mechanical stimulation of the hind paw skin. The SNR 
markedly decreased from 0 to 7 days after the implant and remained unchanged during the follow-up (Fig. 6 B, C), without differences between both groups at any time point. However, dexamethasone treatment resulted in higher number of active sites from which afferent nerve signals could be recorded during follow-up, yet not significantly ( $p>0.05$, Kruskal-Wallis test, Fig.6D).

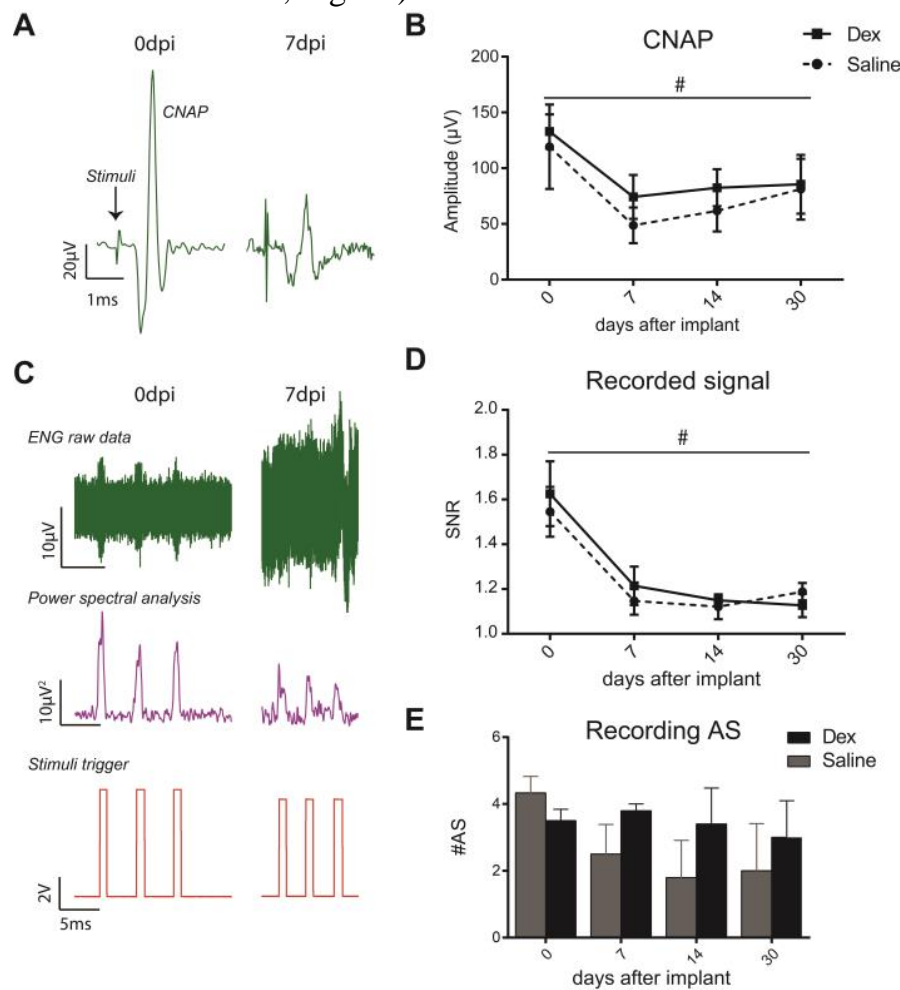

Figure 6. Recording of nerve signals with TIME. (A) Representative CNAP recordings after 0 and 7 days of implantation (dpi). (B) Maximum CNAP amplitude recorded from active sites in TIMEs implanted in the sciatic nerve of saline and dexamethasone treated rats. (C) Representative neural activity recordings after mechanical stimuli in the paw at 0 and 7 days of implantation. (D) Signal-to-noise (SNR) ratio over time in recordings made with TIMEs implanted in saline or dexamethasone treated rats. (E) Number of active sites (AS) from which recording of nerve signals was obtained with a SNR above 1.01 over time. Two-way ANOVA, \#p<0.05 vs time.

\section{Nerve histology}

The histological evaluation of TIMEs implanted after 3 months showed that the TIMEs were within the sciatic nerve, traversing peroneal and tibial fascicles (Fig. 7A, B). Although axons were still close to the active sites, the electrodes were surrounded by a thick capsule of fibrotic tissue, as shown by the hematoxylin-eosin staining in samples taken after 3 months of implantation. The capsule thickness was slightly lower in dexamethasone-treated rats $(54 \pm 18 \mu \mathrm{m})$ than in the saline rats $(74 \pm 13 \mu \mathrm{m})($ Fig. $7 \mathrm{C})$. Finally, foreign body giant cells (FBGCs) were present in close contact with the polyimide substrate, but without significant differences in number between groups (Fig. 7D).
A

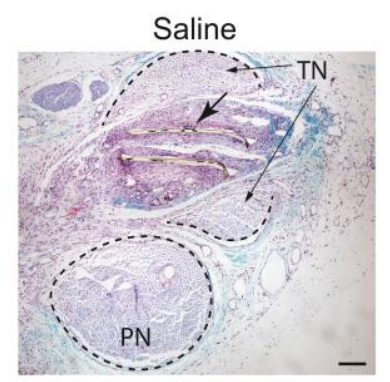

C

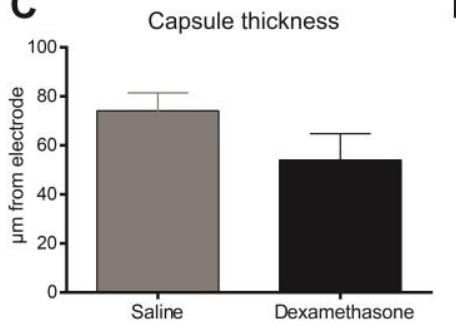

B

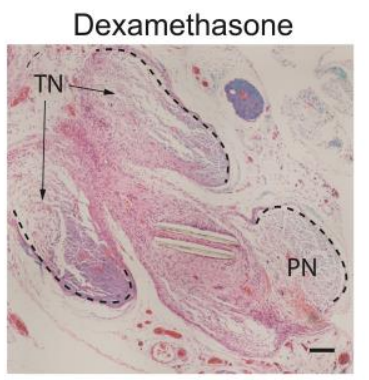

D

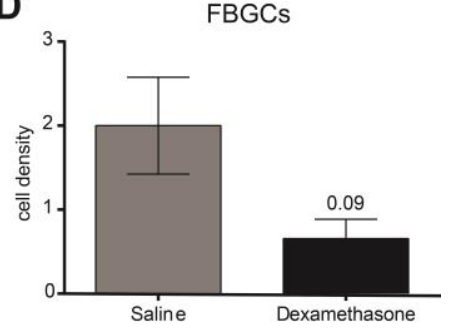

Figure 7. Histological transverse nerve sections stained with LFB-HE showing TIMEs implanted in the sciatic nerve of rats after 12 weeks with (A) saline or (B) dexamethasone treatment. Dotted lines delimit nerve fascicles: $\mathrm{TN}=$ tibial nerve, $\mathrm{PN}=$ peroneal nerve. Arrow in A points to active sites in the implanted electrode. Scale bars in (A-B) $150 \mu \mathrm{m}$. (C) Quantification of the thickness of the capsule formed around the electrode in saline and dexamethasone treated groups. (D) Number of FBGCs around the surface of the implanted electrodes in each studied group. T-test vs saline-treated group.

\section{DISCUSSION}

Neural electrodes are a key component of interfaces for the communication between the nervous system and bionic prostheses. Despite the considerable advances achieved in biomaterial and electrode designs [1], [35], improved stability is desired to maintain the functionality of implanted electrodes over long periods of time. One of the factors that contribute to the failure or decline of function of neural implants is the FBR [9]. The encapsulation of the implanted devices enlarges the distance between the axons membrane and the active sites and also increases the tissue resistance [36], resulting in an increase in the current needed to stimulate the axons and also a decrease of the SNR during recordings. This study combines two of the most promising strategies to improve the long-term functionality of neural implants in peripheral nerves: transversal and flexible intraneural electrodes (i.e., TIME) and anti-inflammatory treatment (i.e., dexamethasone) by systemic administration.

TIME electrodes have been previously shown to provide good stimulation capabilities in animal models [2], [32] and humans [3], [4]. Although these studies reported adequate values of impedance and injected charge for useful stimulation in human patients, an increase in the stimulation threshold was observed during the first month of implant. The present study is in agreement since the stimulation current needed to activate the nerve increased during the first weeks of implant, followed by stabilization of the threshold values. Moreover, the selectivity indices found in this work are similar to those shown in previous studies with intraneural electrodes [2], [10], [37], confirming the stable selectivity in subchronic implants. However, few works have focused on the recording 
capabilities of this type of electrode and only in acute conditions [32]. Our results on subchronically implanted TIME electrodes show a decrease in the amplitude of the recorded signals, either compound or single action potentials from one week post-implant, although it was stabilized in the following weeks.

Several studies have investigated the effect of antiinflammatory drugs on the FBR to neural implants, with positive effects on electrode functionality and on the amount of FBR [23]-[26]. However, this is the first work in which it has been evaluated with intraneural electrodes in the peripheral nerve. Dexamethasone administration substantially decreased the stimulation intensity needed to activate the nerve during the first month of implant. We have previously described the time course of the FBR to nerve implants, and found that the first phase of the FBR peaks at one month [9], and that dexamethasone treatment decreases the amount of infiltrating macrophages within the nerve and also the thickness of the capsule formed around intraneural devices [28]. In this study, we observed that dexamethasone-treated animals also had a tendency, yet not significant, to present thinner tissue capsule. These changes can be related to the decrease in macrophage infiltration in the nerve tissue, as demonstrated by lower presence of FBGCs after 3 months of implant. Dexamethasone treatment also resulted in better functionality of the implanted TIMEs. In particular, there was less displacement of the electrode within the nerve during the first weeks of implant, confirmed by the maintenance of the active sites stimulation profile in the follow-up testing. This fact may be in disagreement with the reduction of the tissue capsule, since fewer adherences would be expected to be related with greater displacement. However, the antiinflammatory treatment may decrease not only the cell infiltration in the nerve tissue but also the edema after the implantation. This edema and the subsequent changes due to cell infiltration in the saline-treated group may result in weaker adherences and larger electrode motion. Thus, the reduction of the stimulation intensity may be due to the decrease in capsule thickness around TIME implanted in nerves under dexamethasone treatment. In addition to the reduced distance between the active sites and the axons to stimulate, changes in tissue deposition and cellular infiltration may imply changes in the electrical properties of the surrounding tissue. In fact, we found more functioning contacts able to evoke a muscle response in the treated group. Besides, less motion of the electrode within the nerve would imply more steady stimulation and recording in subchronic implants. This is of particular interest since improved bidirectional control of upper limb prostheses is based on stimulating specific motor axons and on decoding nerve signals using featured-detection algorithms [3], [32], [38]. Thus, stable electrodes within the peripheral nerves offer more steady signals in subchronic implants. It is worth noting that the impact of electrode motion and of tethering forces are much larger in rats compared to humans due to the electrodenerve size relationship, adding challenges for the resistance of electrodes, wires and connectors, which are often among the reasons for the loss of functional contacts over time of implantation, as previously reported [10]. Indeed, we minimized the fixation of the TIME to the rat nerve, compared to the surgical procedure used in human implants [3] or the implant of SELINE electrodes in rats [10] that secure a stable position, in order to assess the effect of intraneural motion.

A limitation of the present study is the lack of evaluation of the electrode itself with e.g. impedance analysis. It is true that neural interfaces can loss its functional capabilities not only due to biological causes but material degradation or active sites corrosion. Further experiments should consider the analysis of these parameters. Nevertheless, the reduction of FBR with dexamethasone treatment yielded better results with the electrodes over time.

On the other hand, dexamethasone treatment did not improve the capability for recording neural signals. The quality of the recorded signal relies on the amplitude of that signal and the placement of the active site with respect to the activated axons. Since the amplitude of nerve signals is on the scale of microvolts, the distance between the axons and the active site and the capsule tissue characteristics become critical to have a good SNR. While the FBR adds about $30 \mu \mathrm{m}$ of separation between the active sites and the axons in longitudinal implants [9], in transversal implants the FBR adds about $60 \mu \mathrm{m}$ of tissue capsule, which would explain the rapid decay in electrode recording capability. In addition, the fibrotic capsule formed around the electrode will presumably offer a higher resistivity, that might be comparable to that of the perineurium [39]. Even though, probably due to the decrease in capsule thickness produced by dexamethasone treatment ( $75 \mu \mathrm{m}$ vs $55 \mu \mathrm{m})$, there were more active sites able of recording small nerve signals.

\section{CONCLUSION}

Overall, our results show a positive effect of dexamethasone treatment on the capability of intraneural electrodes to stimulate nerve axons during the first month of implant. This is the critical time window during which FBR develops and electrodes show the most important shift in threshold charge and impedance [10], [28]. Other previously proposed strategies try to deliver different drugs locally on the implant site. However, these strategies have some limitations in the case of intraneural electrodes, such as the nerve size, since local delivery systems are difficult to adapt to intraneural thin electrodes. Our results demonstrate that systemic administration of anti-inflammatory drugs may be useful and easy to apply. The dosage used in this work, after translation to human equivalent dose (HED) [40] is:

$$
\mathrm{HED}=0.20 \mathrm{mg} / \mathrm{kg} * 6 / 37=0.032 \mathrm{mg} / \mathrm{kg}
$$

which is equal to $2.27 \mathrm{mg} / \mathrm{day}$ for a $70 \mathrm{~kg}$ adult subject. While this dose lies within the range of inflammatory diseases treatments [41], possible side-effects should be monitored for long-term administration. Since dexamethasone is reducing the cellular infiltration during the first month [28], coinciding with the improvement in electrode functionality, short-term administration can be considered for future experiments. Moreover, stronger effects could be expected in more stable 
models of electrode implantation, such those observed in brain implants [25], or in larger nerves (i.e., human implants), since the size mismatch between rat nerves and the implanted electrodes is more prominent than in larger species.

\section{ACKNOWLEDGMENT}

This research was supported by the European Union FPTICT projects NEBIAS (contract number FP7-611687), EPIONE (FP7-602547), FEDER and by the European Union's Horizon 2020 Graphene Flagship Core Project 2 (grant agreement 785219) and FLAG-ERA JTC 2017 project GRAFIN, by TERCEL (RD12/0019/0011) and CIBERNED (CB06/05/1105) funds from the Instituto de Salud Carlos III of Spain, and by Fundación Ramón Areces (CIVP18A3897). The ICN2 is supported by the Severo Ochoa program from Spanish MINECO (Grant SEV-2013-0295), and by the CERCA Programme/Generalitat de Catalunya. The authors thank Monica Espejo and Jessica Jaramillo for technical help.

\section{REFERENCES}

[1] X. Navarro, T. B. Krueger, N. Lago, S. Micera, T. Stieglitz, and P. Dario, "A critical review of interfaces with the peripheral nervous system for the control of neuroprostheses and hybrid bionic systems.," $J$. Peripher. Nerv. Syst., vol. 10, no. 3, pp. 229-58, Sep. 2005.

[2] J. Badia, T. Boretius, D. Andreu, C. Azevedo-Coste, T. Stieglitz, and X. Navarro, "Comparative analysis of transverse intrafascicular multichannel, longitudinal intrafascicular and multipolar cuff electrodes for the selective stimulation of nerve fascicles.," J. Neural Eng., vol. 8, no. 3, p. 036023, 2011.

[3] S. Raspopovic et al., "Restoring Natural Sensory Feedback in Real-Time Bidirectional Hand Prostheses," Sci. Transl. Med., vol. 6, no. 222, p. 222ra19-222ra19, 2014.

[4] C. M. Oddo et al., "Intraneural stimulation elicits discrimination of textural features by artificial fingertip in intact and amputee humans," Elife, vol. 5, pp. 1-27, 2016.

[5] P. M. Rossini et al., "Double nerve intraneural interface implant on a human amputee for robotic hand control," Clin. Neurophysiol., vol. 121, no. 5, pp. 777-783, 2010.

[6] J. M. Anderson, A. Rodriguez, and D. T. Chang, "Foreign body reaction to biomaterials," Seminars in Immunology, vol. 20, no. 6. pp. 86-100, 2008.

[7] K. W. Ward, "A review of the foreign-body response to subcutaneously-implanted devices: the role of macrophages and cytokines in biofouling and fibrosis.," J. diabetes Sci. Technol., vol. 2, no. 5, pp. 768-777, 2008.

[8] M. B. Christensen, S. M. Pearce, N. M. Ledbetter, D. J. Warren, G. A. Clark, and P. A. Tresco, "The foreign body response to the Utah Slant Electrode Array in the cat sciatic nerve," Acta Biomater., vol. 10, no. 11, pp.
4650-4660, 2014.

[9] N. de la Oliva, X. Navarro, and J. del Valle, "Time course study of long-term biocompatibility and foreign body reaction to intraneural polyimide-based implants," J. Biomed. Mater. Res. Part A, vol. 106, no. 3, pp. 746-757, Mar. 2018.

[10] S. Wurth et al., "Long-term usability and biointegration of polyimide-based intra-neural stimulating electrodes," Biomaterials, vol. 122, pp. 114-129, Apr. 2017.

[11] N. de la Oliva, M. Mueller, T. Stieglitz, X. Navarro, and J. del Valle, "On the use of Parylene C polymer as substrate for peripheral nerve electrodes," Sci. Rep., vol. 8, no. 1, p. 5965, Dec. 2018.

[12] T. S. Davis et al., "Restoring motor control and sensory feedback in people with upper extremity amputations using arrays of 96 microelectrodes implanted in the median and ulnar nerves," J. Neural Eng., vol. 13, no. 3, p. 036001, 2016.

[13] Y. Kim and M. I. Romero-Ortega, "Material considerations for peripheral nerve interfacing," $M R S$ Bull., vol. 37, no. 6, pp. 573-581, 2012.

[14] J. L. Skousen, M. J. Bridge, and P. a Tresco, “A strategy to passively reduce neuroinflammation surrounding devices implanted chronically in brain tissue by manipulating device surface permeability.," Biomaterials, vol. 36, pp. 33-43, Oct. 2014.

[15] S. Sommakia, H. C. Lee, J. Gaire, and K. J. Otto, "Materials approaches for modulating neural tissue responses to implanted microelectrodes through mechanical and biochemical means," Curr. Opin. Solid State Mater. Sci., vol. 18, no. 6, pp. 319-328, 2014.

[16] M. Mueller, N. De, and I. Delgado, "Rapid prototyping of flexible intrafascicular electrode arrays by picosecond laser structuring," pp. 1-15.

[17] A. Balaji et al., "Prospects of common biomolecules as coating substances for polymeric biomaterials," RSC Adv., vol. 5, no. 85, pp. 69660-69679, Aug. 2015.

[18] F. Noorisafa, A. Razmjou, N. Emami, Z.-X. Low, A. H. Korayem, and A. A. Kajani, "Surface modification of polyurethane via creating a biocompatible superhydrophilic nanostructured layer: role of surface chemistry and structure," J. Exp. Nanosci., vol. 8080, no. June, pp. 1-23, Sep. 2016.

[19] S. Chen and M. G. Allen, "Extracellular matrix-based materials for neural interfacing," MRS Bull., vol. 37, no. 06, pp. 606-613, Jun. 2012.

[20] L. W. Norton, H. E. Koschwanez, N. A. Wisniewski, B. Klitzman, and W. M. Reichert, "Vascular endothelial growth factor and dexamethasone release from nonfouling sensor coatings affect the foreign body response," J. Biomed. Mater. Res. - Part A, vol. 81, no. 4, pp. 858-869, Jun. 2007.

[21] M. Kastellorizios, F. Papadimitrakopoulos, and D. J. Burgess, "Multiple tissue response modifiers to promote angiogenesis and prevent the foreign body reaction around subcutaneous implants.," J. Control. Release, vol. 214, pp. 103-11, Sep. 2015. 
[22] A. E. Coutinho and K. E. Chapman, "The antiinflammatory and immunosuppressive effects of glucocorticoids, recent developments and mechanistic insights," Mol. Cell. Endocrinol., vol. 335, no. 1, pp. 2-13, Mar. 2011.

[23] L. Spataro et al., "Dexamethasone treatment reduces astroglia responses to inserted neuroprosthetic devices in rat neocortex," Exp. Neurol., vol. 194, pp. 289-300, 2005.

[24] Y. Zhong and R. V Bellamkonda, "Dexamethasonecoated neural probes elicit attenuated inflammatory response and neuronal loss compared to uncoated neural probes.," Brain Res., vol. 1148, pp. 15-27, May 2007.

[25] C. Boehler et al., "Actively controlled release of Dexamethasone from neural microelectrodes in a chronic in vivo study," Biomaterials, vol. 129, pp. 176-187, 2017.

[26] S. J. Park et al., "Functional nerve cuff electrode with controllable anti-inflammatory drug loading and release by biodegradable nanofibers and hydrogel deposition," Sensors Actuators, B Chem., vol. 215, pp. 133-141, 2015.

[27] J. J. FitzGerald, "Suppression of scarring in peripheral nerve implants by drug elution," J. Neural Eng., vol. 13, no. 2, p. 026006, 2016.

[28] N. De la Oliva, X. Navarro, and J. del Valle, "Dexamethasone Reduces the Foreign Body Reaction to Intraneural Electrode Implants in the Peripheral Nerve of the Rat," Anat. Rec., vol. 301, no. 10, pp. 1722-1733, Oct. 2018.

[29] J. Badia, T. Boretius, A. Pascual-Font, E. Udina, T. Stieglitz, and X. Navarro, "Biocompatibility of Chronically Implanted Transverse Intrafascicular Multichannel Electrode (TIME) in the Rat Sciatic Nerve," IEEE Trans. Biomed. Eng., vol. 58, no. 8, pp. 2324-2332, Aug. 2011.

[30] T. Boretius et al., "A transverse intrafascicular multichannel electrode (TIME) to interface with the peripheral nerve," Biosens. Bioelectron., vol. 26, no. 1, pp. 62-69, 2010.

[31] C. Veraart, W. M. Grill, and J. T. Mortimer, "Selective control of muscle activation with a multipolar nerve cuff electrode," IEEE Trans. Biomed. Eng., vol. 40, no. 7, pp. 640-653, 1993.

[32] J. Badia, S. Raspopovic, J. Carpaneto, S. Micera, and X. Navarro, "Spatial and Functional Selectivity of Peripheral Nerve Signal Recording With the Transversal Intrafascicular Multichannel Electrode (TIME)," IEEE Trans. Neural Syst. Rehabil. Eng., vol. 24, no. 1, pp. 20-27, Jan. 2016.

[33] S. Raspopovic, J. Carpaneto, E. Udina, X. Navarro, and S. Micera, "On the identification of sensory information from mixed nerves by using singlechannel cuff electrodes.," J. Neuroeng. Rehabil., vol. 7, p. 17, 2010.

[34] International Organization for Standardization, Biological evaluation of medical devices, vol. 3. 2009.

[35] A. Boddupalli, L. Zhu, and K. M. Bratlie, "Methods for Implant Acceptance and Wound Healing: Material
Selection and Implant Location Modulate Macrophage and Fibroblast Phenotypes," Adv. Healthc. Mater., vol. 5, no. 20, pp. 2575-2594, Oct. 2016.

[36] S. F. Lempka, S. Miocinovic, M. D. Johnson, J. L. Vitek, and C. C. McIntyre, "In vivo impedance spectroscopy of deep brain stimulation electrodes," $J$. Neural Eng., vol. 6, no. 4, p. 046001, Aug. 2009.

[37] A. Cutrone et al., "A three-dimensional self-opening intraneural peripheral interface (SELINE)," J. Neural Eng., vol. 12, no. 1, p. 016016, 2015.

[38] S. Micera et al., "Decoding information from neural signals recorded using intraneural electrodes: Toward the development of a neurocontrolled hand prosthesis," Proc. IEEE, vol. 98, no. 3, pp. 407-417, 2010.

[39] A. Weerasuriya, R. A. Spangler, S. I. Rapoport, and R. E. Taylor, "AC impedance of the perineurium of the frog sciatic nerve.," Biophys. J., vol. 46, no. 2, pp. 167-74, Aug. 1984.

[40] S. Reagan-Shaw, M. Nihal, and N. Ahmad, "Dose translation from animal to human studies revisited," FASEB J., vol. 22, no. 3, pp. 659-661, Mar. 2008.

[41] M. Vyvey, "Steroids as pain relief adjuvants.," Can. Fam. Physician, vol. 56, no. 12, pp. 1295-7, e415, Dec. 2010. 